Results: Two hundred and fifty-five cases of IgG4-ROD were identified in 434 IgG4-RD patients in this study. IgG4-ROD group had almost equal sex ratio (male to female $0.96: 1$ vs $2.14: 1, P<0.001)$, younger age of disease onset $(51.1 \pm 13.2$ vs $55.5 \pm 13.5, P<0.001)$ and diagnosis $(54.4 \pm 12.6$ vs $56.9 \pm 13.4, P=0.054) \mathrm{com}-$ paring with nlgG4-ROD patients. As compared to nlgG4-ROD group, higher percentage of IgG4-ROD patients met the 2019 American College of Rheumatology/European League Against Rheumatism classification criteria (AECC) for IgG4-RD (89.4\% vs $64.8 \%, P<0.001)$, moreover, IgG4-ROD patients had higher AECC scores $(37.0 \pm 12.9$ vs $27.2 \pm 14.0, P<0.001)$ and IgG4-RD responder index (11.4 \pm 6.0 vs $10.2 \pm 6.3, P=0.045)$. Allergic diseases $(65.5 \%$ vs $26.3 \%, P<0.001)$ and multiorgan involvement [6(4-7) vs $3(2-5), P<0.001]$ were more common in IgG4-ROD group. IgG4-ROD was frequently associated with salivary gland (92.5\% vs $45.3 \%, P<0.01$ ), paranasal sinuses (38.8\% vs $12.8 \%, P<0.01$ ), lung (47.8\% vs $32.4 \%, P<0.01)$ and lymph node $(64.3 \%$ vs $46.4 \%, P<0.01)$ involvement, while retroperitoneal fibrosis $(7.1 \%$ vs $21.2 \%, P<0.01)$ and biliary system lesions $(25.1 \%$ vs $35.2 \%, P<0.05)$ were more common in nlgG4-ROD. IgG4ROD patients had higher serum IgG4 levels [983(409-1838) vs 523(225-1370), $P<0.001]$, IgG4/IgG ratio [0.529(0.272-0.879) vs 0.294(0.143-0.629), $P<0.001$, IgE levels [339.7(160.8-847.6) vs 259.4(83.1-653.3), $P=0.022]$ and lower CRP levels [2.24(1.00-4.70) vs 3.05(1.26-12.45), $P=0.004]$. The initial glucocorticoid plus immunosuppressant was a protective factor for IgG4-ROD relapse (HR $0.279,95 \% \mathrm{Cl} 0.165-0.470, \mathrm{P}<0.001)$, and IgG4-ROD patients treated with initial glucocorticoid plus immunosuppressant had longer relapse-free survival time than patients treated with initial glucocorticoid monotherapy ( 43 vs $17, \mathrm{P}<0.001$ ). Conclusion: IgG4-ROD patients had distinctive clinical features compared with nlgG4-ROD patients. The initial glucocorticoid plus immunosuppressant was a protective factor for IgG4-ROD relapse, which could prolong the relapse-free survival time of IgG4-ROD patients. These findings may have important implications for understanding and management of IgG4-ROD.

Disclosure of Interests: None declared

DOI: 10.1136/annrheumdis-2021-eular.652

\section{AB0759 INDEX OF COMORBIDITY IN PATIENTS WITH LOBULAR PANNICULITIS-LIPODERMATOSCLEROSIS}

O. Egorova ${ }^{1}$, B. Belov ${ }^{1}$, A. Potapova ${ }^{2} .{ }^{1}$ V.A. Nasonova Research Institute of Rheumatology, Laboratory for the Study of Comorbid Infections and Monitoring the Safety of Drug Therapy, Moscow, Russian Federation; ${ }^{2}$ V.A. Nasonova Research Institute of Rheumatology, V.A. Nasonova Research Institute of Rheumatology, Moscow, Russian Federation

Background: Early diagnosis of comorbid burden and treatment of rheumatological patients, especially panniculitis $(\mathrm{Pn})$, is a complex problem, the solution of which lies in an interdisciplinary approach and the development of a general algorithm for managing patients.

Objectives: To study the structure and frequency of comorbid conditions in patients with Pn-lipodermatosclerosis (LDS)

Methods: We examined 53 patients ( 3 men and 50 women aged 18 to 80 ) with verified LDS, who were observed at the V.A. Nasonova Research Institute of Rheumatology for ten years on average. The duration of the disease varied from 2 weeks to 20 years. Clinical, laboratory and instrumental examination of patients was carried out twice a year. Clinical examination was carried out to determine localization, prevalence, color and number of the affected areas of skin and subcutaneous fat (SCF), as well as pain intensity according to the Visual Analogue Scale (VAS). Laboratory and instrumental research included standard blood and urine tests, as well as computed tomography of the chest and Doppler ultrasound of the lower extremities. To assess the relationship between the presence of comorbid pathology and the course of LDS the patients were analyzed with the CIRS and Charlson indices.

Results: Most patients were overweigh women (60.3\%) with average weight of $91.5 \pm 21.8 \mathrm{~kg}$. Based on the duration of the disease we identified the main variants of the course of the disease: acute (up to 3 months), subacute (from 3 to 6 months), and chronic (more than 6 months). Skin lesions were associated with polyarthralgias $(n=18)$ and/or myalgias $(n=12)$, mainly in the area of the affected limb. In 16 patients an increase in the erythrocyte sedimentation rate was recorded on average up to $23.8 \pm 7.8 \mathrm{~mm} / \mathrm{h}$. The level of C-reactive protein (CRP) was more than 3 times higher than normal in 7 patients, 4 of them had an acute course of LDS. In the study group 17 patients did not have comorbid diseases, $64.7 \%$ of them were under 50 years old with an acute course of LDS $(p=0.02)$. Concomitant pathology was detected in $68 \%$ of patients mainly with chronic LDS. In $67.9 \%$ of cases it was presented by chronic venous insufficiency (CVI), in $60.3 \%$ - by exogenous constitutional obesity, in $45.2 \%$ - by rheumatic diseases $(75 \%$ of these patients had osteoarthritis, $17 \%$ - rheumatoid arthritis, $8 \%$ - antiphospholipid syndrome) and in 39.6\% - arterial hypertension. Most patients had one concomitant disease, almost a fifth of patients had two concomitant diseases. The proportion of patients with three comorbid diseases was
$11.1 \%$, four comorbid diseases $-8.3 \%$ and five comorbid diseases $-5.5 \%$. When assessing the Charlson index, 10-year survival rates over $90 \%$ (index values from 0 to 2 points) were registered in $66 \%$ of patients, from 53 to $77 \%$ (index values of $3-4$ ) - in $26.4 \%$ and less than $21 \%$ ( $\geq 5$ points) - in $7.5 \%$. The comorbidity index correlated with the age of patients $(r=0.8 ; p<0.05)$. There was no correlation between the Charlson index and the duration of LDS $(r=0.3 ; p=0.2)$. In patients older than 61 one or more comorbid conditions were recorded. The CIRS index for this group averaged $4.2 \pm 0.3$ points (range $0-10$ ), in most patients $(45.2 \%)$ the CIRS index did not exceed 5 points. An analysis of the relationship between the Charlson and the CIRS scales confirmed their significant correlation at the level of $r=0.5, p=0.0000001$.

Conclusion: In patients with LDS a high incidence of comorbid pathology was detected. The treatment of this variant of panniculitis requires an interdisciplinary approach and interaction between doctors of different specialties.

Disclosure of Interests: None declared

DOI: 10.1136/annrheumdis-2021-eular.861

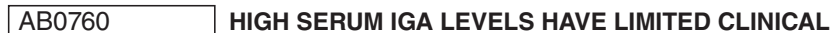 SIGNIFICANCE IN PATIENTS WITH IGG4-RELATED DISEASE DIAGNOSED BY EXPERTS}

S. Tsuge ${ }^{1}$, I. Mizushima ${ }^{1}$, S. Shin ${ }^{1}$, T. Yoshinobu ${ }^{1}$, R. Hoshiba ${ }^{1}$, R. Nishioka ${ }^{1}$, T. Zoshima ${ }^{1}$, S. Hara ${ }^{1}$, Y. Suzuki ${ }^{1}$, K. Ito ${ }^{1}$, M. Kawano ${ }^{1} .{ }^{1}$ Kanazawa University Hospital, Department of Rheumatology, Kanazawa, Japan

Background: While the diagnostic and classification criteria for IgG4-related disease (IgG4-RD) have been recently developed [1-3], it is known that, without appropriate exclusions, some non-IgG4-RDs may meet these criteria. In particular, hyper IL-6 syndromes, including Castleman disease, can be misdiagnosed as IgG4-RD. Some clinical findings, including elevated serum levels of C-reactive protein (CRP) or IgA, have been suggested to be useful for differentiating hyper IL-6 syndromes from IgG4-RD [4]. However, since few clinical studies have focused on IgG4-RD with high serum IgA levels, its clinical significance has not been well known.

Objectives: This study aimed to clarify the clinical significance of high serum IgA levels in patients with IgG4-RD.

Methods: We retrospectively investigated the clinical features of 170 patients with IgG4-RD on the basis of the presence or absence of elevated serum IgA levels ( $>410 \mathrm{mg} / \mathrm{dL}$ ) at the time of diagnosis. The diagnosis of IgG4-RD was made by experts on the basis of the fulfillment of the comprehensive diagnostic criteria and/or each organ-specific diagnostic criteria.

Results: Elevated serum IgA levels were observed in 18 patients (10.6\%). In the patients with elevated serum IgA levels, serum CRP levels were higher (1.14 \pm 1.18 vs. $0.31 \pm 0.63 \mathrm{mg} / \mathrm{dL}, \mathrm{p}=0.003$ ) and the prevalence of relapse during the clinical course was lower $(5.6 \%$ vs. $27.6 \%, p=0.046)$ than in those without elevated serum IgA levels. However, there were no significant differences in the other clinical features including the number of involved organs $(2.4 \pm 1.3 \mathrm{vs}$. $2.8 \pm$ 1.6, $p=0.443$ ) and inclusion scores of the ACR/EULAR classification criteria (32 \pm 14 vs. $36 \pm 17, p=0.374)$. To evaluate the influence of serum IgA elevation on relapse, we performed Cox regression analysis, which showed that the elevated serum IgA levels had no significant association with lower incidence of relapse but a tendency of it (hazard ratio $0.997,95 \%$ confidence interval $0.994-1.000$, $\mathrm{p}=0.055$ ) during the clinical course. In addition, a prompt improvement in the IgG4-RD responder index [5] during the clinical course was seen in the patients with serum IgA elevation, suggesting a similar good response to glucocorticoids as in those without it.

Conclusion: The findings of the present study suggest that IgG4-RD patients with high serum $\lg A$ levels can be diagnosed and treated in the same way as those without it, although they may be characterized by mild serum CRP elevation.

\section{REFERENCES:}

[1] Wallace ZS et al. The 2019 American College of Rheumatology/European League Against Rheumatism classification criteria for IgG4-related disease. Ann Rheum Dis. 2020;79:77-87

[2] Umehara $\mathrm{H}$ et al. The 2020 Revised Comprehensive Diagnostic (RCD) Criteria for IgG4-RD. Mod Rheumatol. 2020 Dec 4:1-14. doi: 10.1080/14397595.2020.1859710. Online ahead of print.

[3] Umehara $\mathrm{H}$ et al. Current approach to the diagnosis of IgG4-related disease - Combination of comprehensive diagnostic and organ - specific criteria. Mod Rheumatol. 2017;27:381-91.

[4] Sato $Y$ et al. Systemic IgG4-related lymphadenopathy: A clinical and pathologic comparison to multicentric Castleman's disease. Mod Pathol.2009;22:589-99.

[5] Carruthers MN et al. Development of an IgG4-RD Responder Index. Int J Rheumatol. 2012;2012:259408.

Disclosure of Interests: None declared

DOI: 10.1136/annrheumdis-2021-eular.944 